\title{
Older Age Significantly Affects Mortality of Patients with Papillary Thyroid Carcinoma Only When They Have High-Risk Features
}

\author{
Yasuhiro Ito $^{1} \cdot$ Akira Miyauchi $^{1} \cdot$ Takuya Higashiyama $^{1} \cdot$ Hiroo Masuoka $^{1} \cdot$ \\ Minoru Kihara ${ }^{1} \cdot$ Akihiro Miya $^{1}$
}

Published online: 24 February 2020

(C) The Author(s) 2020

\begin{abstract}
Background Old age is a known prognostic factor for mortality in patients with papillary thyroid carcinoma (PTC). This study aimed to investigate the relationship between cause-specific survival (CSS) following PTC and the extent of old age.

Methods We enrolled 4692 patients aged $\geq 55$ years with PTC who underwent surgery between 1989 and 2009 at Kuma Hospital (median follow-up period 140 months). The presence of at least one of the following was used to classify the patients as high risk: (1) tumor sizes $>4 \mathrm{~cm}$, (2) node metastasis $\geq 3 \mathrm{~cm}$, (3) significant extrathyroid extension corresponding to T4a, (4) extranodal tumor extension, and (5) distant metastasis. T1N0M0 PTC was classified as low risk, and all other patients were classified as intermediate risk. We divided all patients into three categories based on age: $55-64,65-74$, and $\geq 75$ years.

Results One low-risk patient (0.04\%), 18 intermediate-risk patients (1.5\%), and 105 high-risk patients $(9.4 \%)$ died of PTC, and CSS of high-risk patients was poorer than that of others $(p<0.0001)$. The CSS of low and intermediaterisk patients did not differ with age. However, CSS of high-risk patients became significantly poorer with advancing age ( $p=0.0017$ for 55-64 years vs. 65-74 years, and $p=0.0109$ for 65-74 years vs. $\geq 75$ years).

Conclusions Advanced age has a significant prognostic impact on CSS only for high-risk patients with PTC.
\end{abstract}

\section{Introduction}

Age is one of the most important prognostic factors for cancer-specific mortality in patients with papillary thyroid carcinoma (PTC). In the 8th edition of American Joint Committee on Cancer (AJCC), age cutoff was changed from 45 to 55 years, and patients aged $<55$ years with differentiated thyroid carcinoma (DTC), including those with PTC, were classified as stage I unless distant metastasis (M1) was detected at presentation [1]. Several studies subsequently validated this finding, demonstrating that a

Yasuhiro Ito

ito01@kuma-h.or.jp

1 Department of Surgery, Kuma Hospital, 8-2-35, Shimoyamate-dori, Chuo-ku, Kobe, Hyogo 650-0011, Japan cutoff age at 55 years more accurately predicted the prognosis of patients with PTC than a cutoff age at 45 years [2-4]. However, it remains debatable whether only one cutoff age is adequate for predicting patients' prognoses. Adam et al. showed that in patients with PTC who underwent surgery identified from the SEER database, 10-year cause-specific survival (CSS) rates were excellent for patients $<50$ years (better than 99\%), but became poorer with advanced age: 50-59 years, 98.1\%; 60-69 years, 94.8\%; 70-79 years, 91.5\%; 80-89 years, $79.2 \%$; and $\geq 90$ years, $73.9 \%$ [5]. Furthermore, in a single-institution study by Chereau et al., authors divided their patient series assessing PTC into three categories, young ( $<65$ years), older (65-75 years), and very old patients ( $>75$ years). The results showed that the 5-year CSS rate was $81.3 \%$ for very old patients, which is poorer than that 
for older patients (92.9\%) and for young patients (94.7\%) [6].

The Japan Association of Endocrine Surgery (JAES) has its own risk classification of PTC, which is as follows: very low-risk PTC (T1aNOM0) based on the 8th AJCC TNM classification (low-risk papillary microcarcinoma) [1], lowrisk PTC (T1bN0M0), high-risk PTC, and intermediaterisk PTC-PTC not classified as very low, low, and high risk [7]. Classification as high-risk PTC is based on the presence of at least one of these clinical features: (1) tumor size $(T)>4 \mathrm{~cm}$, (2) lymph node metastasis $(N) \geq 3 \mathrm{~cm}$, (3) significant extrathyroid extension corresponding to T4a, (4) extranodal tumor extension based on intraoperative findings, and (5) presence of M1. We previously investigated the difference in prognosis between patients $\geq 55$ years and those $<55$ years for each risk classification [7]. We showed that very low- or low-risk patients had an excellent prognosis after surgery regardless of age because only two patients died of thyroid carcinoma. CSS of intermediate-risk patients $\geq 55$ years $(15$-year CSS rates $98 \%$ ) was poorer than that of those $<55$ years $(15$-year CSS 100\%), but the difference in CSS rates between these subsets was small. However, for high-risk patients, the 15 -year CSS rate of patients $\geq 55$ years was $85 \%$, which was much lower than that of those $<55$ years at $98 \%$ [7]. These findings indicate that advanced age does not affect the prognosis of patients with PTC uniformly.

To show whether and how advanced age affects prognoses of PTC patients, we analyzed patients aged $\geq 55$ years with PTC and compared CSS among patients with very low- or low-risk, intermediate-risk, and high-risk PTC by subdividing patient age into three categories, 55-64 years, $65-74$ years, and $\geq 75$ years.

\section{Patients and methods}

Between 1989 and 2009, 10,348 patients underwent surgery with R0 or R1 resection for PTC at Kuma Hospital. Of these patients, 4692 aged 55 years or older at the time of surgery and followed up for at least 6 months after surgery were enrolled in this study. They consisted of 678 males and 4014 females. All patients were pathologically diagnosed with PTC, and those with other thyroid malignancies such as follicular carcinoma, medullary carcinoma, anaplastic carcinoma, and thyroid lymphoma were excluded. Of the study sample, 2848 patients underwent total thyroidectomy, and the remaining 1844 underwent other thyroidectomies such as hemithyroidectomy, isthmectomy, and subtotal thyroidectomy. Overall, central node dissection was performed in 4447 patients, and 2309 of these underwent prophylactic or therapeutic, unilateral or bilateral modified radical neck dissection. Fourteen patients also underwent therapeutic upper mediastinal dissection. The median follow-up time after surgery was 140 months (6-336 months). To date, 125 patients underwent radioactive iodine (RAI) administration $\geq 30 \mathrm{mCi}$ for ablation treatment, adjuvant therapy, or cancer therapy.

All patients were followed up with blood examinations and imaging studies such as an ultrasound 1-2 times per year. Chest roentgenography, a computerized tomography scan, and bone scintigraphy were also performed for follow-up assessments at each physician's discretion. For patients who were followed up in other hospitals after surgery, we constantly sent questionnaires to patient residences to ask whether they were alive, died of thyroid carcinoma, or died of other reasons. At the same time, we also asked for the name of attending physicians and hospitals. Besides, we recorded the information about patients' prognoses from the hospitals we referred patients to. The study endpoint is cause-specific survival (CSS).

The classification of patients with PTC into risk groups in our series was subject to the classifications conducted by JAES as described in Introduction. However, we analyzed very low-risk and low-risk patients in a single group, as low-risk patients. Informed consent was obtained from all individual participants included in the study. We sought approval from the ethical committee; however, approval was not required due to the retrospective nature of the study.

Fisher's exact test was used to compare variables. For time-sequence analysis, the Kaplan-Meier method with log-rank tests was utilized using the StatView statistical software. We used Cox proportional hazards models for multivariate analyses. $p$ values $<0.05$ were considered statistically significant, and values $0.05 \leq p<0.1$ were considered marginally significant. For the multivariate analysis, we included factors with a statistically or marginally significant prognostic value from univariate analysis.

\section{Results}

Table 1 shows the relationship between clinicopathological features and patient age at surgery. The incidence of highrisk features such as distant metastasis (M1) $(p=0.0011)$, tumor size $(T)>4 \mathrm{~cm}(p<0.0001)$, extrathyroid extension (Ex) positive $(p<0.0001)$, and extranodal tumor extension (LNEx) positive $(p=0.0279)$ significantly increased with advanced age. Regarding clinical node metastasis $(\mathrm{N})$, the incidence of patients with $N \geq 3 \mathrm{~cm}$ was significantly higher in patients aged $\geq 65$ years than that in those aged 55-64 years $(p<0.0001)$, although the incidence did not significantly differ between patients aged $65-74$ years and those aged $\geq 75$ years. 
Table 1 Relationship between clinicopathological features and the three age groups

\begin{tabular}{|c|c|c|c|c|c|}
\hline Variables & Age $55-64$ years & Age $65-74$ years & Age $\geq 75$ years & Total & $p$ values \\
\hline \multicolumn{6}{|l|}{$M$ factor } \\
\hline$M 1$ & $20(0.8 \%)$ & $20(1.3 \%)$ & $13(2.6 \%)$ & $53(1.1 \%)$ & \multirow[t]{2}{*}{0.0011} \\
\hline$M 0$ & $2644(99.2 \%)$ & $1510(98.7 \%)$ & $445(97.4 \%)$ & $4639(98.9 \%)$ & \\
\hline \multicolumn{6}{|l|}{$T$} \\
\hline$>4 \mathrm{~cm}$ & $193(7.2 \%)$ & $147(9.6 \%)$ & $67(13.5 \%)$ & $407(8.7 \%)$ & \multirow[t]{2}{*}{$<0.0001$} \\
\hline$\leq 4 \mathrm{~cm}$ & $2471(92.8 \%)$ & $1383(90.4 \%)$ & $431(86.5 \%)$ & $4285(91.3 \%)$ & \\
\hline \multicolumn{6}{|l|}{ Ex } \\
\hline Positive & $329(12.3 \%)$ & $294(19.2 \%)$ & $151(30.3 \%)$ & $774(16.5 \%)$ & \multirow[t]{2}{*}{$<0.0001$} \\
\hline Negative & $2335(87.7 \%)$ & $1236(80.8 \%)$ & $347(69.7 \%)$ & $3918(83.5 \%)$ & \\
\hline \multicolumn{6}{|l|}{$N$} \\
\hline$\geq 3 \mathrm{~cm}$ & $59(2.2 \%)$ & $121(7.9 \%)$ & $24(4.8 \%)$ & $204(4.3 \%)$ & \multirow{3}{*}{$<0.0001^{\mathrm{a}}$} \\
\hline$<3 \mathrm{~cm}$ & $527(19.8 \%)$ & $324(21.2 \%)$ & $121(24.3 \%)$ & $972(20.7 \%)$ & \\
\hline Negative & $2078(78.0 \%)$ & $1085(70.8 \%)$ & $353(70.8 \%)$ & $3516(75.0 \%)$ & \\
\hline \multicolumn{6}{|l|}{ LNEx } \\
\hline Positive & $44(1.7 \%)$ & $35(2.3 \%)$ & $17(3.4 \%)$ & $96(2.0 \%)$ & \multirow[t]{2}{*}{0.0279} \\
\hline Negative & $2620(98.3 \%)$ & $1495(97.7 \%)$ & $481(96.6 \%)$ & $4596(98.0 \%)$ & \\
\hline \multicolumn{6}{|l|}{ Thyroidectomy } \\
\hline Total & $1627(61.0 \%)$ & $938(61.3 \%)$ & $283(56.8 \%)$ & $2848(60.7 \%)$ & \multirow[t]{2}{*}{0.1718} \\
\hline Non-total & $1037(39.0 \%)$ & $582(38.7 \%)$ & $215(43.2 \%)$ & $1844(39.3 \%)$ & \\
\hline \multicolumn{6}{|l|}{ Node dissection } \\
\hline $\mathrm{CND}+\mathrm{MND}$ & $1330(49.9 \%)$ & $758(49.5 \%)$ & $221(44.3 \%)$ & $2309(49.2 \%)$ & \multirow[t]{3}{*}{0.0719} \\
\hline CND only or not done & $1334(50.1 \%)$ & $772(50.5 \%)$ & $277(55.7 \%)$ & $2383(50.8 \%)$ & \\
\hline Total & $2664(100 \%)$ & $1530(100 \%)$ & $498(100 \%)$ & $4692(100 \%)$ & \\
\hline
\end{tabular}

$T$ Tumor size, $L N E x$ extranodal tumor extension on intraoperative findings, $N$ clinical node metastasis, Ex significant extrathyroid extension on intraoperative findings, $C N D$ central node dissection, $M N D$ modified radical neck dissection

${ }^{\mathrm{a}} N \geq 3 \mathrm{~cm}$ versus others and age 55-64 years versus others

No significant difference could be observed for the extent of thyroidectomy and lymph node dissection in the three subsets. However, the incidences of total thyroidectomy and modified radical neck dissection were slightly lower in patients $\geq 75$ years than in others. In our study, the numbers of low-, intermediate-, and high-risk patients were 2371,1205 , and 1116, respectively. Table 2 shows the incidence of low-, intermediate-, and high-risk patients in $55-64$ years, $65-74$ years, and $\geq 75$ years age groups. The incidence of high-risk cases significantly increased with advanced age: $19.6 \%$ for patients aged 55-64 years, $26.1 \%$ for those aged $65-74$ years, and $39.2 \%$ for those aged $\geq 75$ years $(p<0.0001)$. Of 1116 high-risk patients, $888(79.6 \%)$ underwent total thyroidectomy. The incidence of total thyroidectomy for high-risk patients in the three subsets was $82.0 \%$ (427 of 521 patients), $81.5 \%$ (326 of 400 patients), and $69.2 \%$ (135 of 195 patients) for the 55-64 years, 65-74 years, and $\geq 75$ years age groups, respectively. This indicates that the incidence of total thyroidectomy in high-risk patients $\geq 75$ years was

Table 2 Relationship between risk classification and patient age

\begin{tabular}{|c|c|c|c|c|}
\hline Age (years) & High risk & Intermediate risk & Low risk & Total \\
\hline $55-64$ & $521(19.6 \%)$ & $706(26.5 \%)$ & $1437(53.9 \%)$ & $2664(100 \%)$ \\
\hline $65-74$ & $400(26.1 \%)$ & $382(25.0 \%)$ & $748(48.9 \%)$ & $1530(100 \%)$ \\
\hline$\geq 75$ & $195(39.2 \%)$ & $117(23.5 \%)$ & $186(37.3 \%)$ & $498(100 \%)$ \\
\hline Total & $1116(23.8 \%)$ & $1205(25.7 \%)$ & $2371(50.5 \%)$ & $4692(100 \%)$ \\
\hline
\end{tabular}

$p<0.0001$, high risk versus intermediate risk and low risk 


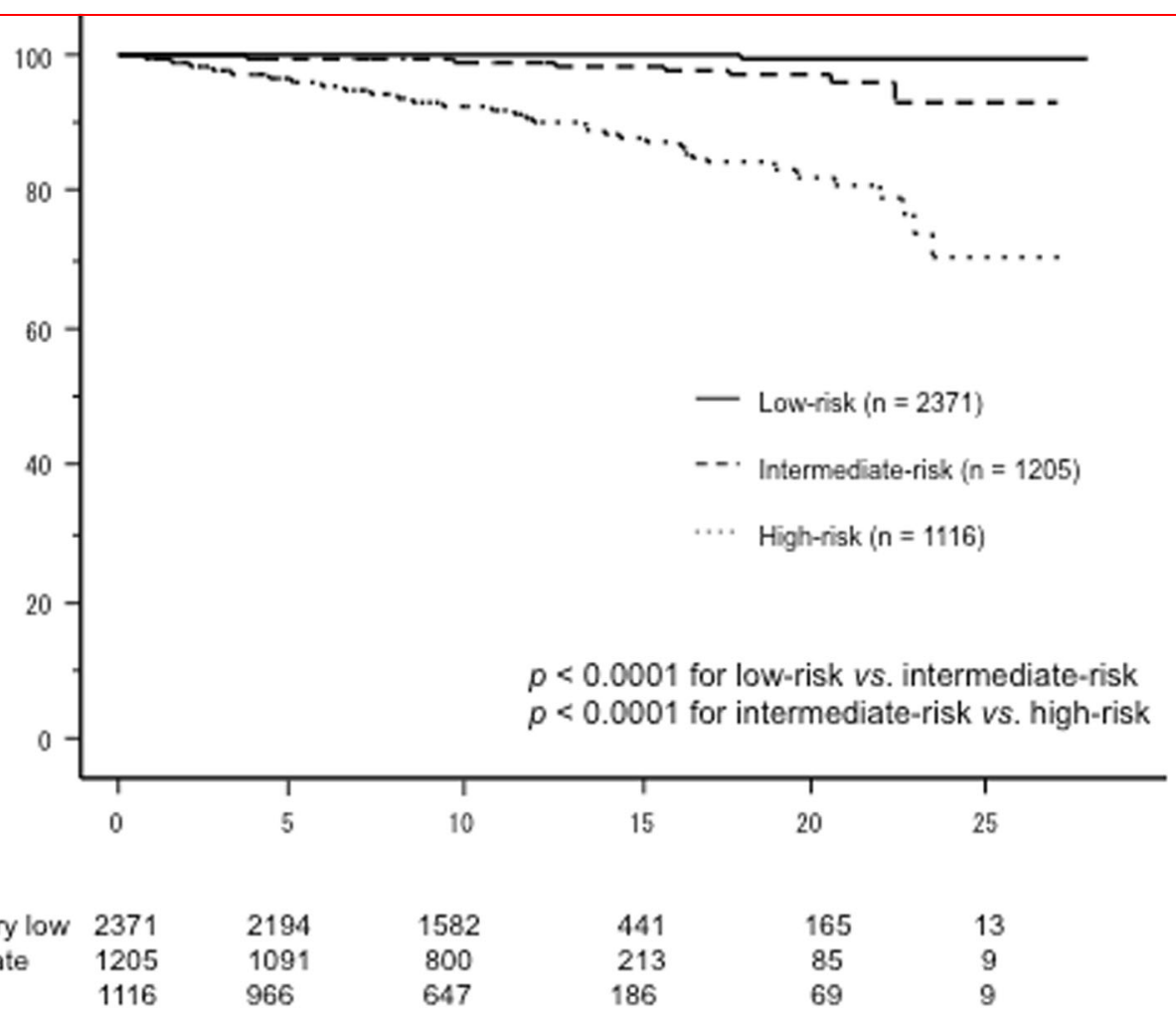

Fig. 1 Kaplan-Meier curves for CSS of low-, intermediate-, and high-risk patients aged $\geq 55$ years

significantly lower $(p<0.0001)$ than that for those aged 55-74 years, which might be related to physicians performing less extensive surgeries for patients $\geq 75$ years due to old age.

Next, we analyzed the difference in the CSS of low-, intermediate-, and high-risk patients with PTC aged $\geq 55$ years. The incidence of cancer mortality in high-risk patients (105 patients, 9.4\%) was much higher than in intermediate-risk patients (18 patients, $1.5 \%)$ and low-risk patients (1 patient, 0.04\%). Figure 1 shows the KaplanMeier curves for three subsets of patients. CSS was progressively and significantly poorer from low- to high-risk patients $(p<0.0001$ for low-risk vs. intermediate-risk patients, and $p<0.0001$ for intermediate-risk vs. high-risk patients). The 10-year, 15-year, and 20-year CSS rates were $100 \%, 100 \%$, and $99.6 \%$ for low-risk, $98.9 \%, 98.3 \%$, and $97.1 \%$ for intermediate-risk, and $92.4 \%, 87.7 \%$, and $82.1 \%$ for high-risk patients, respectively.

Since only one low-risk patient died of thyroid carcinoma, we performed further subset analyses only for intermediate-risk and high-risk patients. Figure 2a shows the Kaplan-Meier curves for intermediate-risk patients aged 55-64, 65-74, and $\geq 75$ years. The 10-year, 15-year, and 20-year CSS rates were $98.9 \%, 98.4 \%$, and $96.8 \%$ for patients aged $55-64$ years, $99.1 \%, 98.2 \%$, and $98.2 \%$ for those aged $65-74$ years, and $99.0 \%, 99.0 \%$, and $99.0 \%$ for those aged $\geq 75$ years, respectively. The CSS of these three subsets showed no significant difference $(p=0.7004$ for patients aged 55-64 years vs. those aged 65-74 years, and $p=0.8966$ for those aged $65-74$ years vs. those aged $\geq 75$ years).

As shown in Fig. 2b, the CSS of high-risk patients was progressively poorer with advanced age $(p=0.0017$ for patients aged 55-64 years vs. those aged 65-74 years, and $p=0.0109$ for patients aged $65-74$ years vs. those aged $\geq 75$ years). The 10-year, 15-year, and 20-year CSS rates were $95.2 \%, 92.7 \%$, and $88.6 \%$ for patients aged 55-64 years, $93.0 \%, 85.1 \%$, and $72.7 \%$ for those aged 65-74 years, and $81.2 \%, 70.7 \%$, and not defined (none of the patients underwent follow-up for 20 years) for those aged $\geq 75$ years, respectively. The Kaplan-Meier curves for patients aged $65-74$ years and those $\geq 75$ years crossed over after 20 years of follow-up, which may be because of the small number of patients at risk followed up for longer than 20 years.

Other than patient age, M1 $(p<0.0001), N>3 \mathrm{~cm}$ $(p<0.0001)$, and LNEx positive $(p<0.0001)$ showed a significant prognostic value for CSS of high-risk patients, while Ex-positive $(p=0.0500)$ had marginal significance in univariate analysis. However, $T>4 \mathrm{~cm}$ did not have 


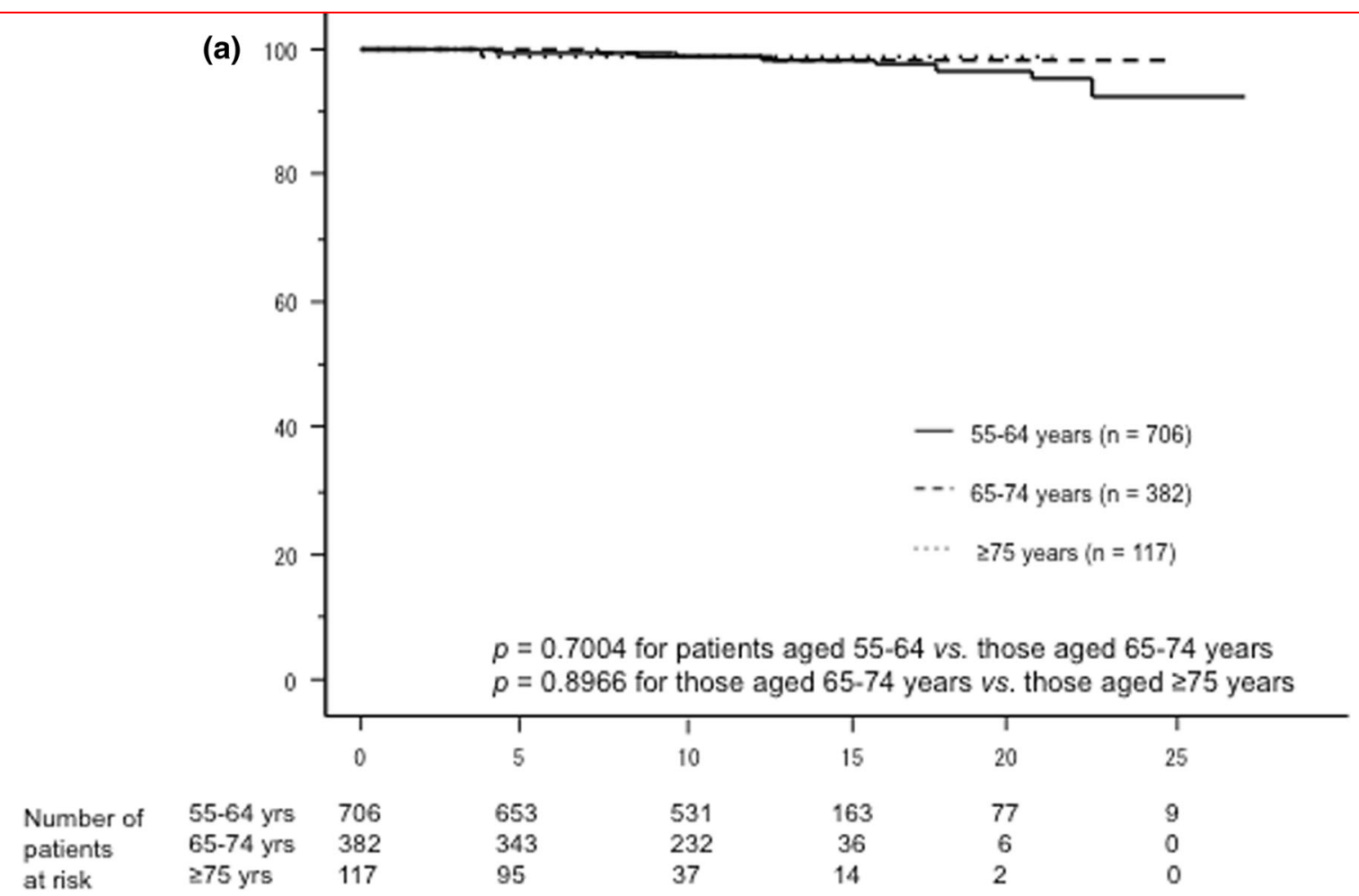

(b)

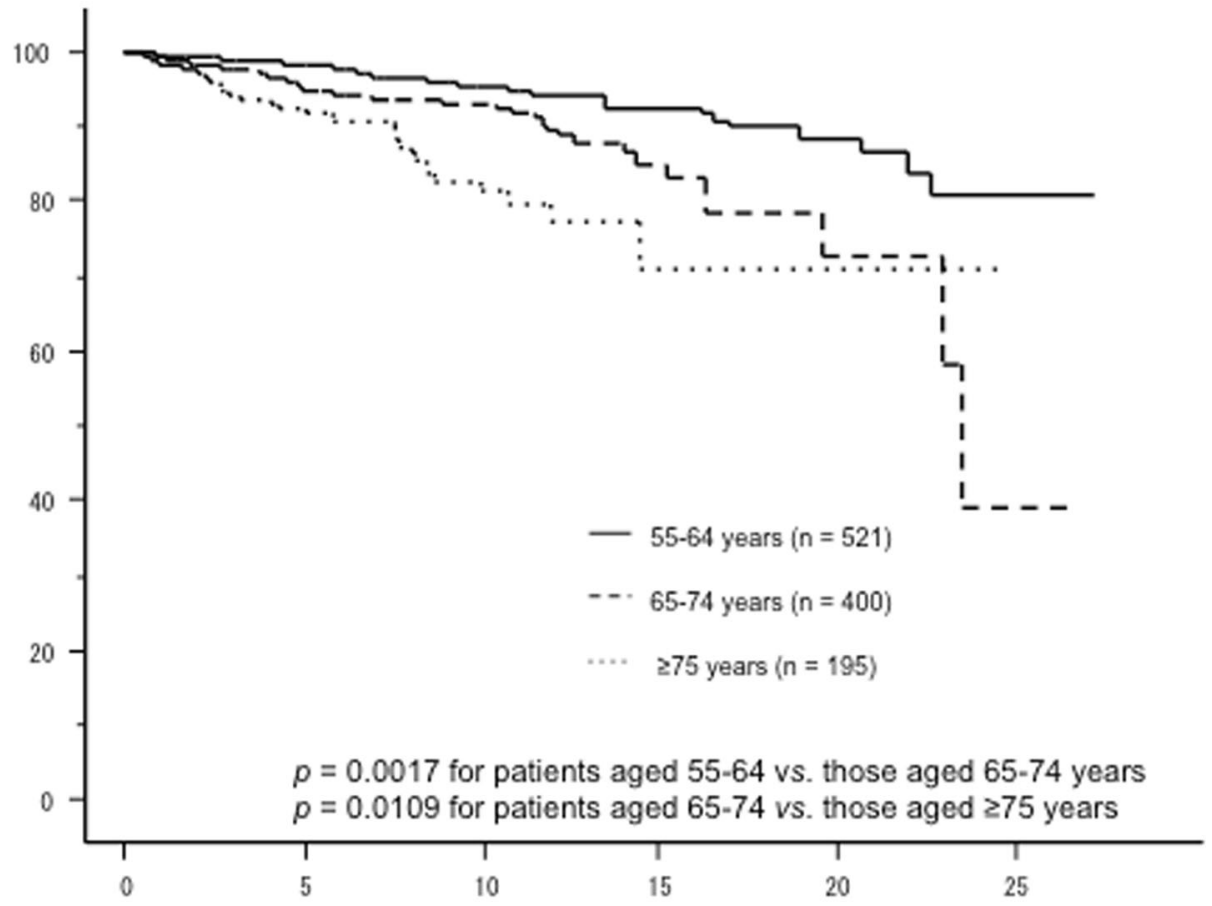

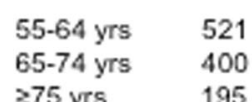

$\geq 75$ yrs
195

483
339
144

483
339
144

382
209
56

56

125
51
10

$\begin{array}{cc}52 & 7 \\ 12 & 2 \\ 5 & 0\end{array}$

Fig. 2 a Kaplan-Meier curves for CSS of intermediate-risk patients aged 55-64 years, 65-74 years, and $\geq 75$ years. b Kaplan-Meier curves for CSS of high-risk patients aged 55-64 years, 65-74 years, and $\geq 75$ years 
any prognostic impact $(p=0.2395)$. On multivariate analysis for the factors having prognostic significance on univariate analysis, all except for LNEx-positive had prognostic significance (Table 3). The hazard ratio for M1 was the highest at 9.6154, and that for those aged $\geq 75$ years showed the second highest value at 3.3898. The hazard ratio of patients aged 65-74 was 1.7483 .

We investigated the prognostic significance of clinicopathological factors for patients $\geq 75$ years. We found that only M1 $(p<0.0001)$ had a prognostic value on univariate analysis, and thus, we did not perform a multivariate analysis.

Since this study includes patients who underwent surgery in long periods, from the 1980s to 2000s, we divided high-risk patients into two categories: 511 patients who underwent surgery after February 2005 (Group A) and 605 patients who underwent surgery before then (Group B). In both groups, CSS of patients was significantly or tended to be poorer with advanced age $(p=0.06721$ for patients $\geq 75$ years vs. $65-74$ years and $p=0.00285$ for patients 65-74 years vs. 55-64 years in Group A, and $p=0.0040$ for patients $\geq 75$ years vs. $65-74$ years and $p=0.00037$ for patients aged 65-74 years vs. 55-64 years in Group B).

\section{Discussion}

In this study, we demonstrated that in patients $\geq 55$ years with PTC, (1) the incidence of high-risk PTC significantly increased with advanced age, (2) CSS of low-risk patients was excellent regardless of patients' age, (3) CSS of intermediate-risk patients did not differ according to patient age, and (4) CSS of high-risk patients significantly became poorer with advanced age.

The observed increased incidence of high-risk patients with advancing age in this study is consistent with the

Table 3 Multivariate analysis of prognostic factors for patients aged $\geq 55$ years with PTC

\begin{tabular}{lrl}
\hline Variables & $p$ values & Hazard ratio $(95 \% \mathrm{CI})$ \\
\hline Age 65-74 years & 0.0174 & $1.7483(1.1038-2.7701)$ \\
Age $\geq 75$ years & $<0.0001$ & $3.3898(2.0492-5.5866)$ \\
$M 1$ & $<.0001$ & $9.6154(5.9172-15.6250)$ \\
Ex positive & 0.0113 & $1.8116(1.1442-2.8736)$ \\
$N \geq 3 \mathrm{~cm}$ & $<0.0001$ & $2.7548(1.7241-4.4248)$ \\
LNEx positive & 0.0683 & $1.7065(0.9606-30.3030)$
\end{tabular}

$C I$ Confidence interval, LNEx extranodal tumor extension on intraoperative findings, $N$ clinical node metastasis, $E x$ significant extrathyroid extension on intraoperative findings, $M 1$ distant metastasis at diagnosis results of a previous study by Chereau et al. [6] who reported that patients $>75$ years were more likely to have advanced TNM stage, greater tumor size, and a greater number of tumors and extracapsular invasion than those aged 75 years or younger. This may be because old patients have less opportunity of receiving medical checkup and their PTCs are more likely to be detected after progression or because PTC in old patients is more likely to turn into high risk than in young patients.

Previous studies have shown that the prognosis of older patients with PTC was not uniform but became poorer with advancing age. Adam et al. showed that the prognosis of patients with PTC in old age became poorer in a linear fashion [5]. Also, Chereau et al. demonstrated that CSS of patients $>75$ years with PTC was significantly poorer than those aged $65-75$ years and aged $<65$ years [6]. In this study, however, we showed that only one patient $(0.04 \%)$ with very low-risk PTC and 18 patients $(1.5 \%)$ with intermediate-risk PTC died of thyroid carcinoma, indicating that carcinoma death is a rare event for older patients with PTC without high-risk features. In addition, these prognoses did not significantly change with advancing age of patients. In contrast, 105 out of 1116 patients (9.4\%) of high-risk PTC died of thyroid carcinoma, and the prognosis of high-risk patients became significantly poorer with advancing age. These findings strongly suggest that advanced age can significantly affect the prognosis of only high-risk patients with PTC, which sharply contrasts with findings of previous studies [5, 6]. The reason for this phenomenon remains unknown. One possibility is that high-risk PTC in old patients has a significantly different tumor biology from that in young patients, while low- and intermediate-risk PTCs do not. Further studies are needed to solve this issue.

Based on our findings, we can conclude that therapeutic strategies do not need to change for older low-risk and intermediate-risk patients. However, how we treat older high-risk patients is an important issue. Because of older age, physicians often choose less extensive therapies. In our series, the incidence of total thyroidectomy for highrisk patients $\geq 75$ years was only $69.2 \%$, which was significantly lower than that in younger patients: $82.0 \%$ and $81.5 \%$ for those aged 55-64 years and those aged 65-74 years, respectively. However, according to the investigation by The Japanese Ministry of Health, Labour and Welfare in 2015, the average life expectancy of humans aged 75, 80, and 85 were rather long, at 15.64, 11.71, and 8.3 years for females and 12.03, 8.83, and 6.22 years for males, respectively [8]. In addition, according to National Comprehensive Cancer Network guidelines in the USA, the upper (top 25th percentile) and middle (50th percentile) quartiles of life expectancy for humans aged 75 years were 17.6 years and 12.6 years for 
women and 15.3 years and 10.4 years for men, respectively [9]. Therefore, active treatment should be considered for high-risk older age patients if they are otherwise healthy and have a good performance status. Recently, molecular target therapies such as sorafenib and lenvatinib were adopted as a line of therapy for RAI-refractory recurrence/metastasis of PTC $[10,11]$. It has been reported that lenvatinib prolonged not only progression-free survival but also overall survival for patients $\geq 65$ years [12]. Therefore, if circumstances allow, high-risk older age patients can be considered for total thyroidectomy with therapeutic/prophylactic lymph node dissection, RAI administration for remnant ablation, adjuvant therapy, or cancer therapy. If metastasis or recurrent lesions are RAIrefractory and progressive, molecular targeted therapy could be considered for patients with a good general condition.

This study has some limitations. First, it is a retrospective study, and the therapeutic strategies including the extent of thyroidectomy and lymph node dissection obtainable at that time significantly differ from those available now. Because of the limited capacity of RAI administration, the number of patients in our series who were administrated RAI $\geq 30 \mathrm{mCi}$ for ablation, adjuvant therapy, and therapy for metastases was much smaller than what would be indicated now. Many patients underwent RAI scintigraphy using a smaller amount of RAI (3-13 mCi), instead. Another important limitation of this study is that we could not present the postoperative course and incidence of surgical complications such as transient and permanent recurrent laryngeal nerve paralysis and hypoparathyroidism. This factor is very important to decide therapeutic strategies for old patients. To date, optimal active therapeutic strategies have not been identified or established, including the extent of surgical treatment, RAI therapy, and, if indicated, molecular targeted therapy that can substantially improve prognoses of high-risk aged PTC patients. This issue requires further studies in consideration of various factors, including surgical complications.

In summary, we demonstrated that for patients aged $\geq 55$ years with PTC, advancing age has the significant prognostic impact of CSS only for high-risk patients. If they are otherwise healthy and have a good general condition, active therapies, including total thyroidectomy, appropriate lymph node dissection, and RAI administration for remnant ablation, adjuvant therapy, or cancer therapy even for those $\geq 75$ years, should be considered, because their life expectancy is longer than what we think. If metastatic/recurrent lesions are RAI-refractory and progressive, treatment for molecularly targeted medicine could be regarded as a successive strategy.
Open Access This article is licensed under a Creative Commons Attribution 4.0 International License, which permits use, sharing, adaptation, distribution and reproduction in any medium or format, as long as you give appropriate credit to the original author(s) and the source, provide a link to the Creative Commons licence, and indicate if changes were made. The images or other third party material in this article are included in the article's Creative Commons licence, unless indicated otherwise in a credit line to the material. If material is not included in the article's Creative Commons licence and your intended use is not permitted by statutory regulation or exceeds the permitted use, you will need to obtain permission directly from the copyright holder. To view a copy of this licence, visit http://creativecommons. org/licenses/by/4.0/.

\section{References}

1. Tuttle RM, Morris LF, Haugen B et al (2017) Thyroid-differentiated and anaplastic carcinoma. In: Amin MB, Edge SB, Greene F, et al. (eds) AJCC cancer staging manual, 8th edn. Springer, New York City

2. Ito Y, Ichihara K, Masuoka $\mathrm{H}$ et al (2010) Establishment of an intraoperative staging system (iStage) by improving UICC TNM classification system for papillary thyroid carcinoma. World $\mathrm{J}$ Surg 34:2570-2580. https://doi.org/10.1007/s00268-010-0710-2

3. Nixon IJ, Kuk D, Wreesmann V et al (2016) Defining a valid age cutoff in staging of well-differentiated thyroid cancer. Ann Surg Oncol 23:410-415

4. Mazurat A, Torroni A, Hendrickson-Rebizant $\mathrm{J}$ et al The age factor in survival of a population cohort of well-differentiated thyroid cancer. Endocr Connect 2:154-160

5. Adam MA, Thomas S, Hyslop T et al (2016) Exploring the relationship between patient age and cancer specific survival in papillary thyroid cancer: rethinking current staging systems. J Clin Oncol 34:4415-4420

6. Chereau N, Tresallet C, Noullet S et al (2016) Prognosis of papillary thyroid carcinoma in elderly patients after thyroid resection. Medicine 95:e5450

7. Ito Y, Miyauchi A, Oda H et al (2019) Appropriateness of the revised Japanese guidelines' risk classification for the prognosis of papillary thyroid carcinoma: a retrospective analysis of 5,845 papillary thyroid carcinoma patients. Endocr J 66:127-134

8. Investigation of the number of patients in 2015, The Ministry of Health, Labour and Welfare, Japan [Internet]. [cited 2019 Oct 23], Available from: https://www.mhlw.go.jp/toukei/saikin/hw/ jinkou/suikei15/

9. NCCN Clinical Practice Guidelines in Oncology (NCCN Guidelines) for Older Adult Oncology Version 1.2019

10. Brose MS, Nutting CM, Jarzab B et al (2014) Sorafenib in radioactive iodine-refractory, locally advanced or metastatic differentiated thyroid cancer: a randomized, double-blind phase 3 trial. Lancet 384:319-328

11. Schlumberger M, Tahara M, Wirth LJ et al (2015) Lenvatinib versus placebo in radioiodine-refractory thyroid cancer. $\mathrm{N}$ Eng J Med 372:621-630

12. Brose MS, Worden FP, Newbold KL et al (2017) Effect of age on the efficacy and safety of lenvatinib in radioiodine-refractory differentiated thyroid cancer in the phase III SELECT trial. J Clin Oncol 35:2692-2699

Publisher's Note Springer Nature remains neutral with regard to jurisdictional claims in published maps and institutional affiliations. 